\title{
Vortex induced vibrations of rectangular cylinders arranged on a grid
}

\author{
F. Rigo ${ }^{1 *}$, V. Denoël ${ }^{2}$ and T. Andrianne ${ }^{1}$ \\ ${ }^{1}$ Wind Tunnel Lab, Dept. of Aerospace and Mechanical Engineering, University of Liège, Belgium, \\ ${ }^{2}$ Stochastic and Structural Dynamics, Dept. of Urban and Environmental Engineering, University of \\ Liège, Belgium \\ *Corresponding author: F. Rigo, francois.rigo@uliege.be
}

\begin{abstract}
A grid arrangement made of one hundred rectangular cylinders fixed to the facade of a house generates strong and disturbing mono-harmonic noise. The cross-flow vibration of the rectangular cylinders is identified as the origin of the noise. The present article proposes a complete investigation of the Vortex Induced Vibration (VIV) combined with a grid effect. It is based on in situ measurements, numerical (finite elements and Computational Fluid Dynamics (CFD)) and extensive wind tunnel (WT) modelling. A comparison between unsteady pressure measurements and CFD results allows to understand the vortex shedding process and synchronization type depending on the wind incidence and spacing of the cylinders. On the basis of this multi- approach parametric investigation, a deep understanding of the VIV-grid phenomenon enables to propose two mitigation techniques. These techniques are tested and their effectiveness is reported in terms of vibration amplitude and acoustic intensity. Rigo et al. 2018)
\end{abstract}

Keywords: Vortex Induced Vibration, Unsteady, Pressure, Aerodynamics, Computational Fluid Dynamics, Bluff Body, Wind Tunnel, Finite Element Model

\section{Introduction}

A vertical louver structure installed on the facade of a house is composed of aluminium rectangular cylinders with a constant horizontal spacing (Figure 1). This architectural structure is dedicated to isolate the occupants installed on the outdoor terrace from the neighbourhood. The grid was designed based on aesthetic and static loading constraints: it is clamped at four extreme points into the piers of the facade.
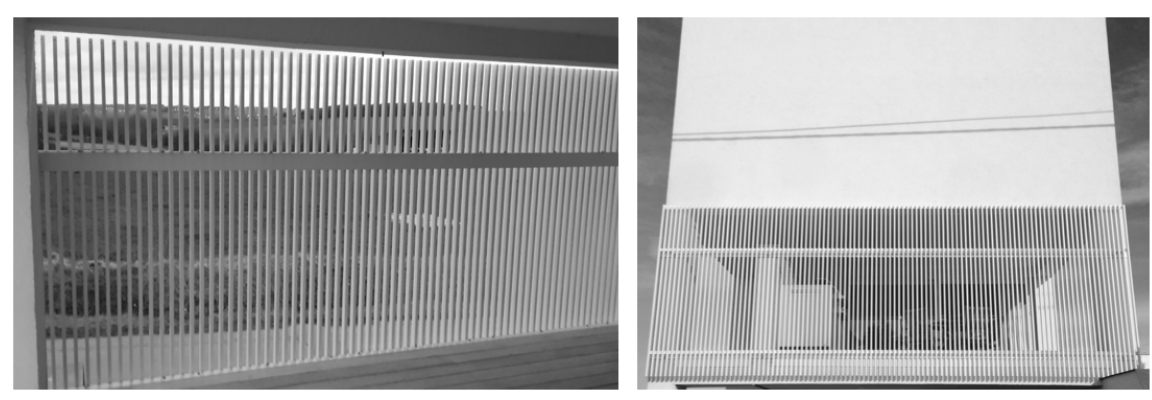

Fig. 1. Real grid on site, from inside (left) and outside (right) 
An intense acoustic emission was reported under specific wind conditions (fall and spring). This undesirable phenomenon creates a characteristic single frequency noise between $50 \mathrm{~Hz}$ and $100 \mathrm{~Hz}$. Due to the large number of rectangular cylinders (100 in total), the phenomenon taking place is similar to a mono-frequency wind instrument creating a strong and unacceptable noise. In addition, vibrations of the louver are transmitted to the structure of the building. On top of this comfort problem, the issue of the safety due to a potential accelerated fatigue degradation of the structure must be considered.

In this paper, a complete analysis of the behaviour of the grid under wind is performed. The objective is first to understand and characterize the aeroelastic instability and then to solve it using an adequate mitigation technique. The starting point is the study of the grid in its initial configuration, then some parameters will be explored, to understand their effects and how they can be changed to solve the problem. The results of the study can be extended to similar structures where the aesthetic objective leading to light and flexible designs must be consistent with external excitations such as atmospheric wind. For that purpose, in situ measurements are performed, to make the link between the vibration of cylinders and the wind conditions (amplitude and direction). Afterwards, a wind tunnel model is developed, based on the real grid, to reproduce real conditions and study other configurations through a parametric analysis. The controlled conditions in laboratory enable to investigate the interaction between wind conditions, vibration and acoustic levels. Finally, numerical methods are used to compare and validate results, by studying structural (modal) properties of the grid on one side (using FEM) and fluid dynamics around the grid on the other (using CFD, with openFoam)

From the initial observations of the structure installed on the house (single frequency, wind direction and amplitude dependencies), the aeroelastic effect responsible of these vibration is the vortex shedding process in the wake of each rectangular cylinder. Hence it consists of a Vortex Induced Vibration (VIV) phenomenon, which results from the interaction between fluid and structural forces, at a given frequency (Techet, 2005, Blevins, 1990). Indeed, observations show a phenomenon which is self-limited, directional and occurs for a certain velocity range $(5 \mathrm{~m} / \mathrm{s}$ to $8 \mathrm{~m} / \mathrm{s})$.

\section{Methodology}

Three complementary approaches are carried out to understand the phenomenon and mitigate the problem: in situ measurements, wind tunnel parametric study on a 1:1 scale model and comparison with numerical models (FE and CFD).

\subsection{In situ measurement}

Experimental measurements are performed on the grid during half a week in windy conditions leading to vibrations of the structure. Anticipating the mitigation technique, two rectangular cylinders were filled with sand. The instrumentation is shown in Figure 2, It consists in

1. Three mono-axis accelerometers (PCB-Piezotronics 3713B112G) are placed at midheight of 3 central cylinders of the grid. The two cylinders filled with sand are instrumented, plus another one empty. These three cylinders are next to each other.

2. An anemometer to measure the wind speed $U$ and direction $\alpha$. The wind components $(u, v)$ are measured in the system of reference of the grid.

3. An acquisition system (computer and internet connection) to remotely acquire data at $640 \mathrm{~Hz}$ 


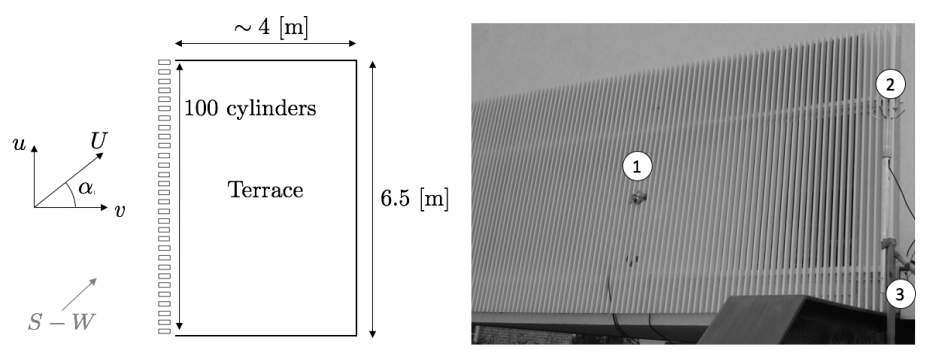

Fig. 2. Grid and terrace geometry, wind components (left) and in situ instrumentation: acclerometers (1), anemometer (2), data logger (3) (right)

The modal properties of the grid are identified experimentally. The grid is made with 100 cylinders of length $2.3 \mathrm{~m}$, with a cross section of $B \times D=5 \mathrm{~cm} \times 2 \mathrm{~cm}$, thickness of $2 \mathrm{~mm}$ and spaced by $T=4 \mathrm{~cm}$. The cylinders are welded on two horizontal supports, vertically spaced by $1.35 \mathrm{~m}$. The welding is equivalent to a pinned support (picture in Figure 2 and schematics in Figure 3. Table 2 shows the eigenfrequencies and damping ratio of the three instrumented cylinders from ambient vibrations. Because of the higher mass of cylinders 1 and 2 filled with sand, the first eigenfrequency is lower than the one of the empty cylinder 3. In addition the sand in cylinders 1 and 2 increases their damping ratio. The Scruton numbers, calculated from the formula proposed in the Eurocode (EC) (introduced in section 1), are presented in this table. The lower value of the third (empty) cylinder, $S c=36.5$, is an indicator that the structure might be affected by VIV (Hansen, 2007).

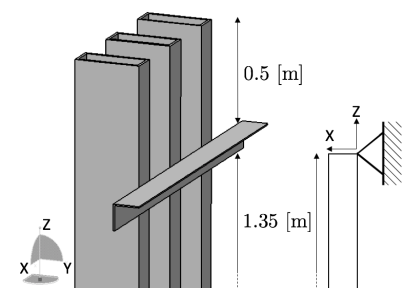

Fig. 3. 3D fixation and boundary condition simplification

\begin{tabular}{c|c|c} 
& $f_{1} \mathrm{~Hz}$ & $f_{2} \mathrm{~Hz}$ \\
\hline Experimental & 59 & 176
\end{tabular}

Table 1: Modal parameters of the grid

\begin{tabular}{c|c|c|c} 
Cylinder & 1 & 2 & 3 \\
\hline$f_{1} \mathrm{~Hz}$ & 44 & 43 & 59 \\
$\zeta \%$ & 1.1 & 0.9 & 0.5 \\
$S c$ from (EC) & 196.7 & 160.9 & 36.5
\end{tabular}

Table 2: Modal characteristics from ambient vibrations of the 3 cylinders instrumented.

\subsection{Wind Tunnel Model}

The wind tunnel model consists in a $1: 1$ scale reproduction of the real grid with 10 cylinders closely spaced and one isolated (Figure 4). Due to height limitations of the test section (max $1.8 \mathrm{~m}$ ), it is decided to limit the height of the cylinders to $1.35 \mathrm{~m}$ (equal to the spacing between the two supporting horizontal beams on site). The 11 rectangular cylinders are connected to a rigid frame with grooves by M3 screws. This allows the horizontal positioning of cylinders 
to study the spacing parameter $T / D$. To guarantee the same fixation type and strength, a setting torque tool is used to screw with a constant torque of $15 \mathrm{Nm}$. The ensemble (Figure (4) is screwed on the turning table, to study the parameter angle of attack $\alpha$.
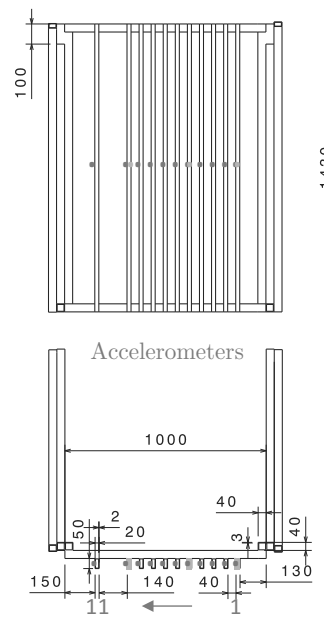
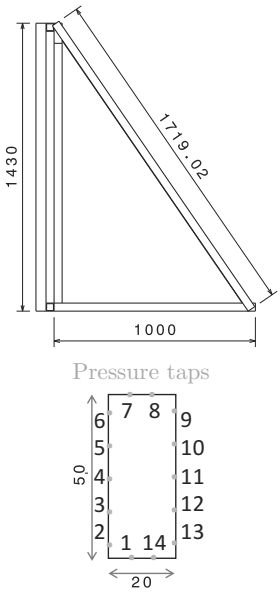

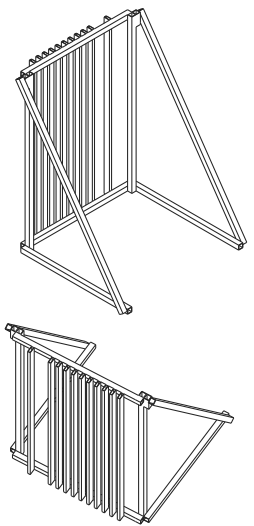

Fig. 4. Drawing of the grid model: top, side, front and axisymmetric views, locations of accelerometers, pressure taps and cylinders numbering, dimensions in $\mathrm{mm}$

The FEM model is used to justify the omission of the upper and lower parts of the cylinders and to retain only the central, bi-pinned part. The same aluminum tubes are used for the design. A comparison between the mode of interest of a single beam $\left(f_{1}=66 \mathrm{~Hz}\right)$ and a FEM model of the real grid $\left(f_{1}=64 \mathrm{~Hz}\right)$ shows very close results. The real grid shows other global mode associated to horizontal frame supports, which consist of simple bending at lower frequency $(f=1.2 \mathrm{~Hz})$ and it does not interact with the flexion mode of the cylinders. The analytic and FEM solutions show that using a beam of $1.35 \mathrm{~m}$ (between pin supports) with or without free ends have the same frequency. Modes associated with the free ends have higher frequencies.

Each cylinder is instrumented at mid-height by a mono-axis accelerometer, measuring the acceleration in the x-direction (see red points in Figure 4). The complete instrumentation diagram together with the dimensions of the model installed in the test section are presented in Figure 5

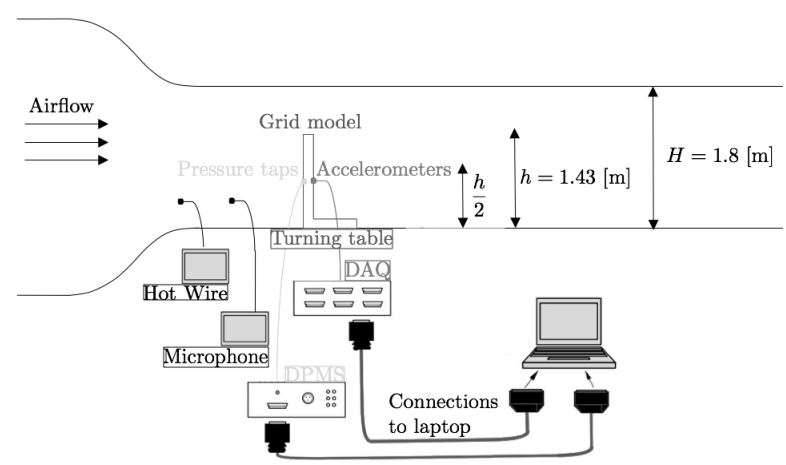

Fig. 5. Wind tunnel model and instrumentation 
Figures 6a and 6b shows the Fast Fourier Transform (FFT) and damping ratios of the mode of interest measured experimentally on every cylinder of the wind tunnel model. The frequency is roughly the same for all cylinders $(f=61 \mathrm{~Hz})$ and very close to the FE prediction $\left(f_{s}^{0}=66\right.$ and $64 \mathrm{~Hz}$ in Table 1 . Damping values are identified using logarithmic decrement. The damping was computed from period to period over the whole time history of the free decay: the mean and the standard deviation of results are reported for each cylinder in Figure $6 \mathrm{~b}$. The damping ratio varies from one cylinder to the other despite the application of the same tightening torque. Note that this situation is probably the same on the real grid, where cylinders have been welded. This modal analysis validates the design of a grid composed of only 10 cylinders (instead of 100), because each cylinders behave structurally as a single beam (very close frequency, around $f_{s}^{0}=61-66 \mathrm{~Hz}$ ).

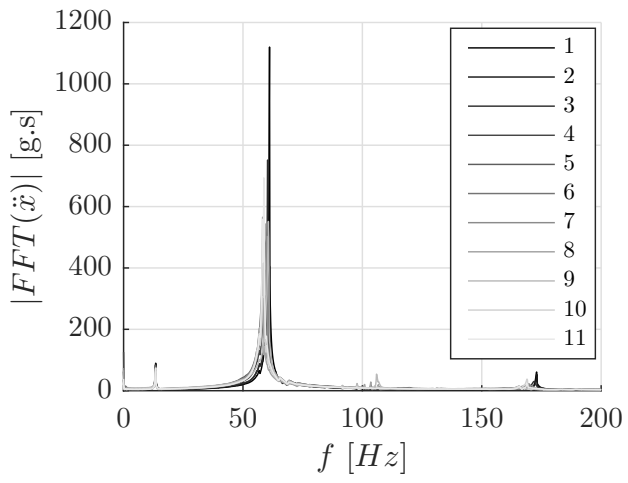

(a) FFT amplitude of cylinders acceleration $\ddot{x}$

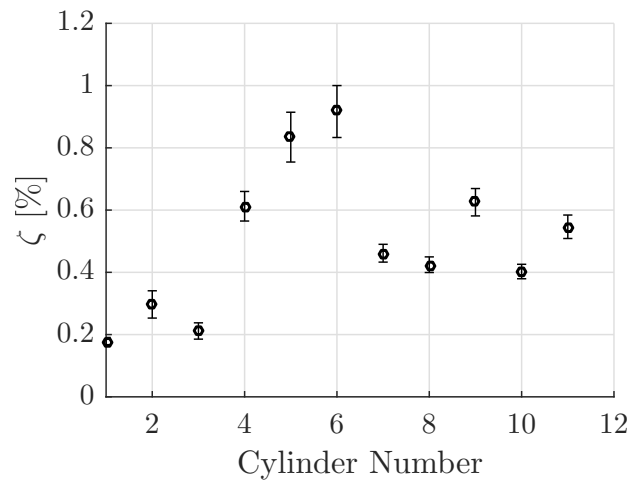

(b) Damping ratio $\zeta$ of cylinders

Fig. 6. Experimental properties of wind tunnel model at wind-off conditions, free decay using hammer excitation

Unsteady pressure measurements are carried out on the surface of the cylinders. Five pressure taps are located along each cylinder chord $B$ and two along cross flow dimension $D$, thus 14 in total (light points in Figures 4 and 57. These taps are connected to a pressure scanner system (DPMS series by Turbulent Flow Instrumentation) by urethane tubes ( $L=1.3 \mathrm{~m}$ and $I D=1.37 \mathrm{~mm})$. The objective is to study the vortex shedding generation and interaction (phase between vortex of two adjacent cylinders). Recorded pressure signals are dynamically calibrated thanks in the frequency domain using the theory by Bergh and Tijdeman (Bergh and Tijdeman, 1967). For both fluid measurements, the sampling frequency is set to $500 \mathrm{~Hz}$. The wind tunnel turbulence intensity is $0.2 \%$ and the blockage of the model is $9.5 \%$.

\subsection{Computational Fluid Dynamics}

A CFD model of the grid is implemented in OpenFoam. An Unsteady Reynolds Averaged Navier-Stokes (URANS) turbulent flow is implemented using a $k-\varepsilon$ turbulence model and wall functions. The objective is to identify the unsteady characteristics of the flow (Strouhal number and force amplitudes) due to the vortex shedding process in grid configuration. The cylinders are static in the fluid domain. The size of the computational domain $L \times W=$ $48 D \times 32.5 D$. The boundary conditions of the fluid domain: at inlet (fixed constant velocity $U$, zero pressure gradient), at outlet (zero velocity gradient, fixed pressure $p=0$ ), at cylinder wall (no slip: zero velocity and zero pressure gradient), up-down sides (slip: zero velocitypressure gradient). Turbulent quantities are computed with OpenFoam functions, using the following initial conditions for inlet: turbulent intensity $I_{u}=\frac{U_{1}}{U}=0.8 \%$ and turbulent length scale $L / D=0.0045$ (Ong, 2013). 


\section{Results}

\subsection{In situ measurements}

As stated previously, two cylinders were filled with sand to increase their mass and damping, hence increasing the Scruton number. The responses of the filled cylinders are compared to the one of an empty (non-modified) cylinder. Figure 7 shows the maximal acceleration for the three accelerometers together with the wind intensity $U$ and direction $\alpha$ during the entire measurement campaign (78 hours). It is observed that each vibration event (peak rms acceleration) happens when the wind speed is higher than $5 \mathrm{~m} / \mathrm{s}$, with a direction varying between $0^{\circ}$ and $50^{\circ}$. The dominant wind direction in Belgium is the South-West and the average intensity is between $4 \mathrm{~m} / \mathrm{s}$ and $5 \mathrm{~m} / \mathrm{s}$. Due to the orientation of the louver, the wind sector $0^{\circ}-50^{\circ}$ with a wind speed of $5 \mathrm{~m} / \mathrm{s}$ is most likely to occur leading to frequent VIV event.
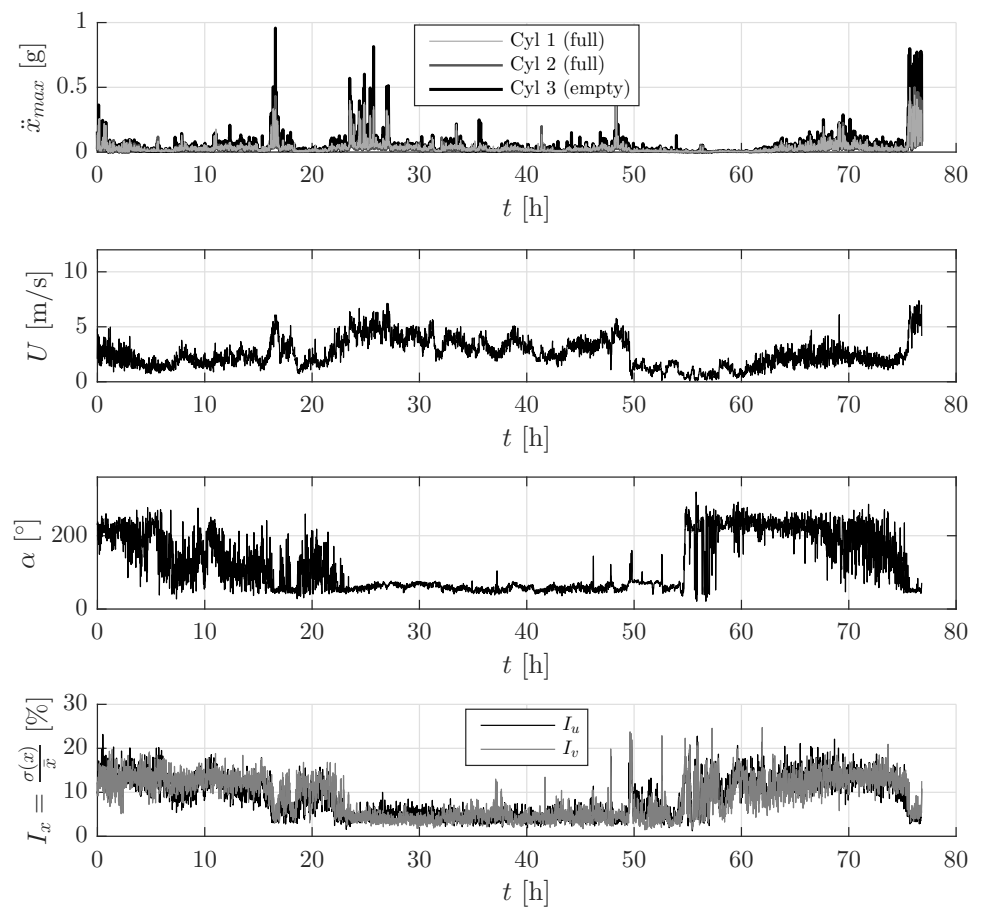

Fig. 7. In situ measurement: peak response during 7 half days, correlation event $-U-\alpha$

On the basis of these first observations, the aeroelastic phenomenon taking place is Vortex Induced Vibration (VIV). This single degree of freedom instability occurs at low wind speed in a given lock-in range. The critical velocity will be quantified in the following sections. The other single degree of freedom in cross-flow, namely transverse galloping, is rejected as responsible for these vibrations because the corresponding quasi-steady critical wind speed is $17.2 \mathrm{~m} / \mathrm{s}$. Note that this value corresponds to an estimation taking into account a structural damping of $0.5 \%$ (from table 2) and a slope of the lift coefficient $A=\frac{d C_{L}}{d \alpha}+C_{D}=-5$ (calculated from the pressure measurements on the grid, see figure 13). This wind speed has never been reached during the in-situ measurement, as can be seen in Figure 7 .

It is proposed to study more precisely the strongest VIV event, taking place between $t=$ $75-78$ hours. Figure 8 shows the vibration envelope $\ddot{x}_{r m s}$ for each cylinders as a function of wind speed and associated wind direction. This figure summarizes the correlation between 
VIV events as a function the corresponding wind conditions $U-\alpha$, instead of time $t$ only.

The maximum amplitude of filled cylinders is roughly half of the empty one. This observation is in opposition with the VIV models predicting maximum response amplitudes inversely proportional to the Scruton number: $y_{\max } \sim 1 / S c$. On the basis of Table 2, a multiplicative factor of 5 or 6 between the amplitudes of the empty cylinder to the filled ones would be expected. Another important observation concerns the wind speed at which the VIV takes place: for the empty cylinder the calculated critical wind speed is around $5 \mathrm{~m} / \mathrm{s}$ and it should be $3.7 \mathrm{~m} / \mathrm{s}$ for the filled ones. Furthermore, it is observed from the upper plot of Figure 7 that the VIV take place simultaneously, i.e. for the same wind conditions, for the three instrumented cylinders. The explanation lies in the fact that the two filled cylinders are surrounded by 98 empty cylinders. If the wind conditions are met to have VIV on the latter, the resulting vibrations can be transmitted by the horizontal supporting beam and excite the filled cylinders in a seismic manner.
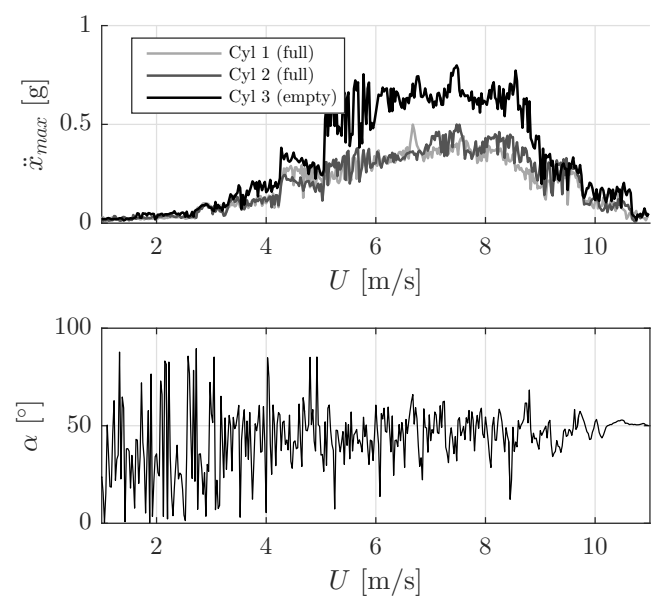

Fig. 8. In situ measurement: VIV curves $\ddot{x}_{r m s}$ envelope for event at $t=77[\mathrm{~h}]$ and corresponding $\alpha$

\subsection{Initial configuration in the wind tunnel}

The wind tunnel model reproducing the geometry and dynamics feature of the grid is tested in controlled wind conditions. In this section we study the effect of the localisation of the cylinder in the grid and the wind direction. Figure 9 shows cylinders response as a function of airspeed for a wind direction $\alpha=0^{\circ}$. Similarly to the in situ measurement, the vibration starts around $U=4-5 \mathrm{~m} / \mathrm{s}$ for all cylinders, having the same frequency (see Figure 6a). Unlike the site measurements, the acceleration reaches higher level (up to $6 g$ here, vs. $1 g$ on site). The comparison between site and laboratory measurements will be presented further. It is observed that the level of vibration is different from cylinder to cylinder. This can be explained by the different damping ratios (see Figure 6b). An alternative manner to present the results is to multiply the rms acceleration by the damping ratio, a damping independent plot can be obtained (see right plot of Figure 9). Cylinders at the center of the grid (4 to 6 , numbering in Figure 4) vibrate the most. They are not affected by end effects, while the amplitude vibration of cylinders 1,2 and 910 at extremities is lower because they are influenced by a non-symmetric flow, even for $\alpha=0^{\circ}$. This observation is due to the grid effect, which is studied in more details in the following sections. 

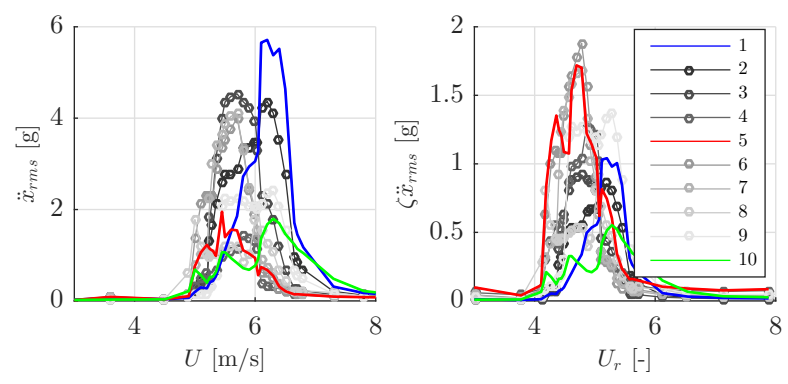

Fig. 9. Wind tunnel model: VIV curves at $\alpha=0^{\circ}$ and $T / D=2,\left(\ddot{x}_{r m s}-U\right)$ and $\left(\zeta \ddot{x}_{r m s}-\right.$ $\left.U_{r}\right)$

\subsection{Parametric studies}

After identifying conditions that lead to VIV instability in situ and in the wind tunnel, a parametric study is performed to study the influence of wind direction and spacing of cylinders. Experimentally, the cylinders are free to vibrate and their acceleration, pressure distribution and wake behaviour are measured and analysed. The CFD results are compared to experimental data to obtain a complete knowledge of the flow around the cylinders. In the CFD model, the cylinders are held statically, so that the effect of the motion of the latters is neglected. This assumption is rather correct because of the small amplitude observed during in situ measurements $(<1 \mathrm{~mm})$. Hence, the CFD model is used to understand the flow, pressure and vortex shedding process.

\subsubsection{Aeroelastic responses}

We focus first on the last two parameters: wind direction $\alpha$ and spacing $T / D$. Figure 10 shows the effect of $\alpha$ and $T / D$ on cylinder response. The following observations are made: (i) increasing the spacing $T / D$ increases the critical reduced velocity at which VIV occurs. (ii) The same tendency holds for the wind direction, hence these two parameters have an effect on the Strouhal number: increasing $T / D$ or $\alpha$ decreases St. (iii) The cylinders located in the center of the grid reach the highest amplitude of vibration for zero and non zero wind direction. These cylinders are affected by a grid effect, amplifying the VIV phenomenon. The acceleration of the flow through the grid alters the flow topology by changing the curvature of the separated shear layers and modifying the vortex shedding process. In the present parametric study, the change of flow topology is due to the grid effect or to the angle of attack. It is traduced by a variation of the Strouhal number. $U_{r}^{\text {crit }}$ is lower and $S t$ appears higher for interfering cylinders than for a single one $\left(S t=0.22\right.$ for $T / D=2$ at $\alpha=0^{\circ}$ whereas $S t=0.17$ for a single cylinder, Figure 12. This enhanced effect by the biased gap flow was previously suggested by Zdravkovich (Zdravkovich, 1985). (iv) When wind direction is around $25^{\circ}$ or $50^{\circ}$, last cylinders end (9-10) are subjected to end effect, lowering the vibration amplitude. Nevertheless, the first ones (1-2-3) in front of the flow are more sollicitated, according to the flow topology in Figure 18.

In addition, an interaction between VIV and galloping is observed in the specific cases of $T / D=3$ and 4 for an angle of attack of $50^{\circ}$. This interaction phenomenon is not studied in the scope of this work which focuses on the particular case of the grid with a spacing $T / D=2$. The parametric analysis of the variation of the Strouhal number with $\alpha$ and $T / D$ can be summarized graphically in Figure 11, where $S t$ is identified from the VIV curves of Figure 10, through $S t=1 / U_{r}^{\text {crit }}$. St decreases when $\alpha$ and $T / D$ increase. The effect of angle of attack is much more marked than spacing. 

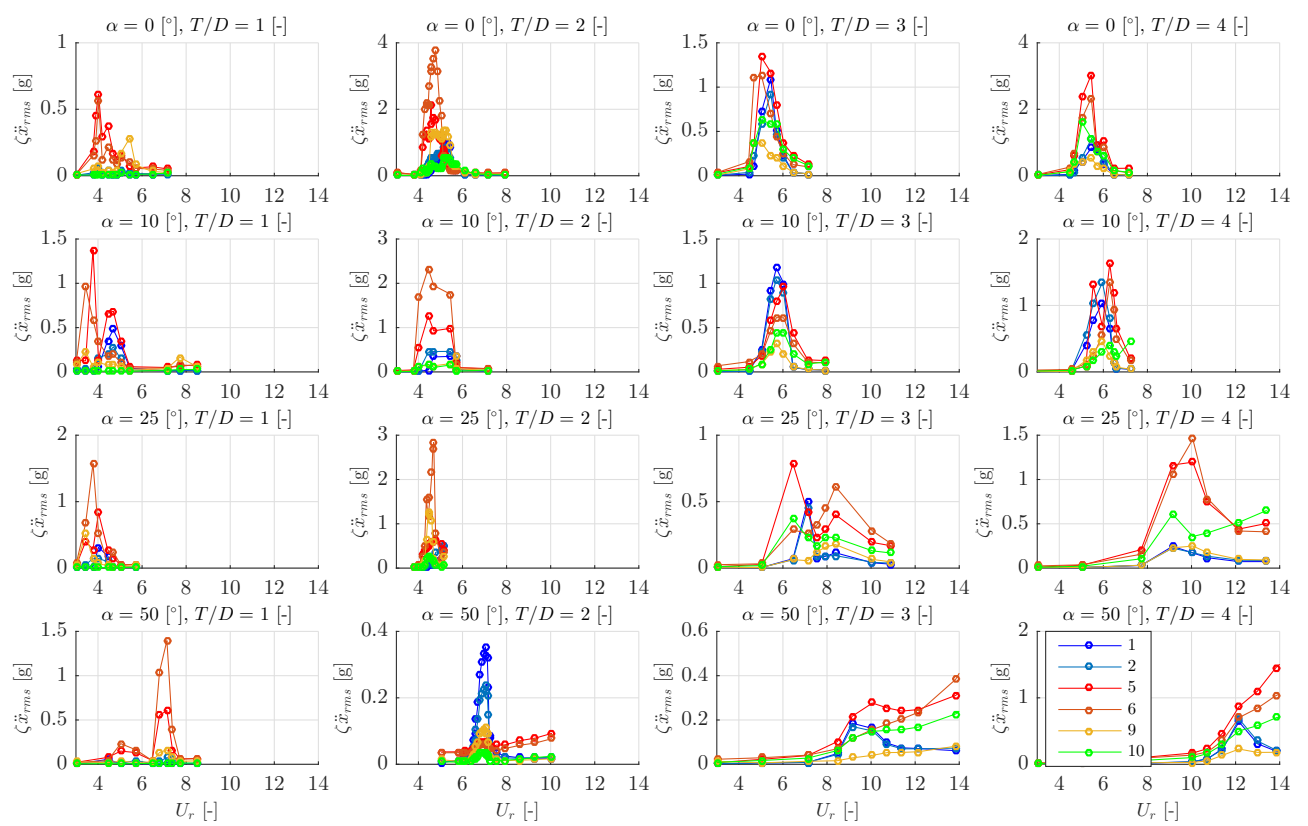

Fig. 10. Wind tunnel model: effect of $\alpha$ and $T / D$ on the wind tunnel model

The grid effect is also presented in Figure 12 where the Strouhal number vs $\alpha$ is plotted for an isolated body and different value of the spacing $T / D=2$. It is observed that the Stouhal number monotonically decreases as a function of the angle of attack unlike the case of an isolated rectangle. In addition, in the case of an isolated cylinder, two values of $S t$ are reported in Figure 12 a high value (0.17) and a low value (0.06). These values were measured by Knisely (Knisely, 1990) who studied the $S t$ number of a rectangular cylinder characterized by different depth to chord ratios. Nakaguchi (Nakaguchi and Muto, 1968) and Okajima (Okajima, 1982) had shown by experiments that for cross sections with $2<B / D<2.8$, a double mode exists in the lift. Physically, it is because of an unsteady reattachment of the separated shear layer on side faces. There are discontinuities in the Strouhal number around $B / D \approx 2.5-2.8$. In the present work, only the high $S t$ value is observed experimentally in the case of a large spacing $(T / D=8$, at $S t=0.18$, see Figure 11$)$. This value is identified because of the velocity range tested. This figure shows than the $S t$ approaches the one for a single cylinder when $T / D$ increases.

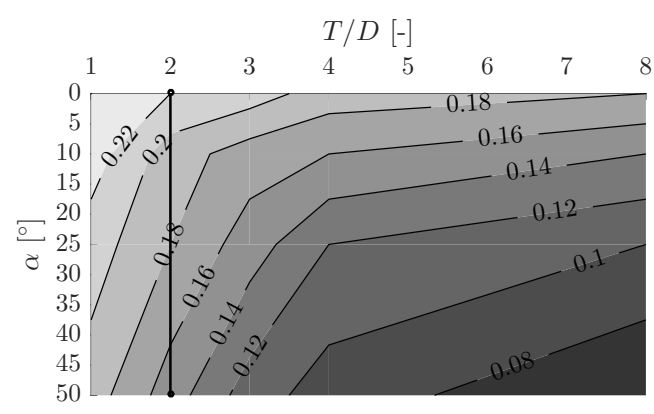

Fig. 11. Wind tunnel model: contour plot of Strouhal number (obtained from VIV response) vs. $\alpha$ and $T / D$, the black line corresponds to the studied grid. 


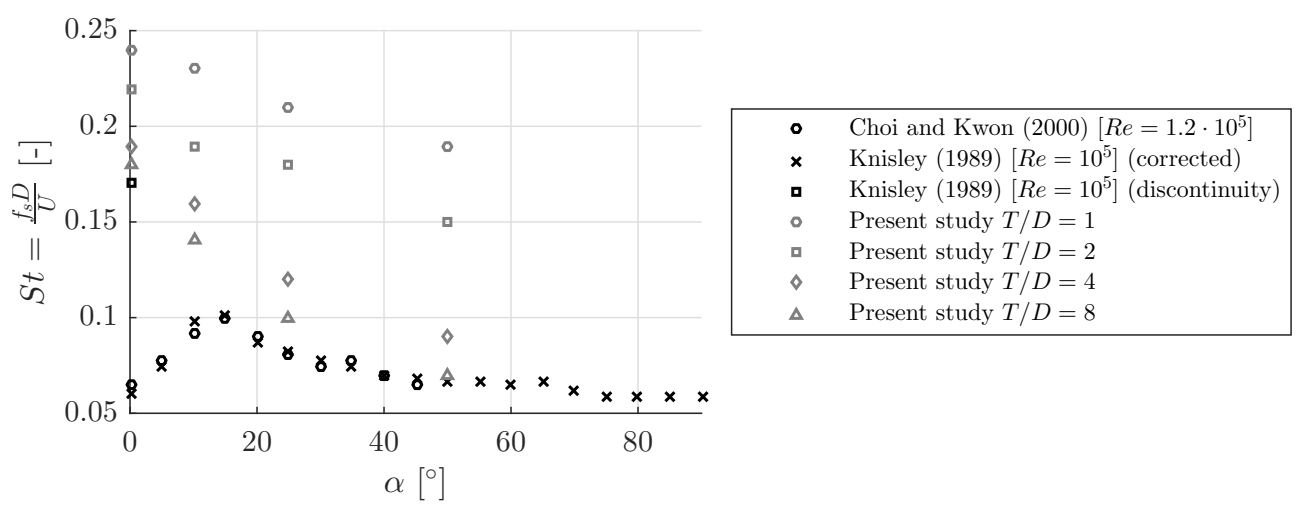

Fig. 12. Strouhal number of a single rectangular cylinder $\left(\frac{B}{D}=2.5\right)$, adapted from Knisely 1990) and (Choi and Kwon, 2000)

\subsubsection{Pressure distributions}

Figure 13 show the mean of the pressure coefficients on cylinders 1,5 and 10 for different values of the spacing parameter $(T / D=1,2,4$ and 8$)$ and wind direction $\left(\alpha=0^{\circ}, 10^{\circ}\right.$ and $\left.50^{\circ}\right)$
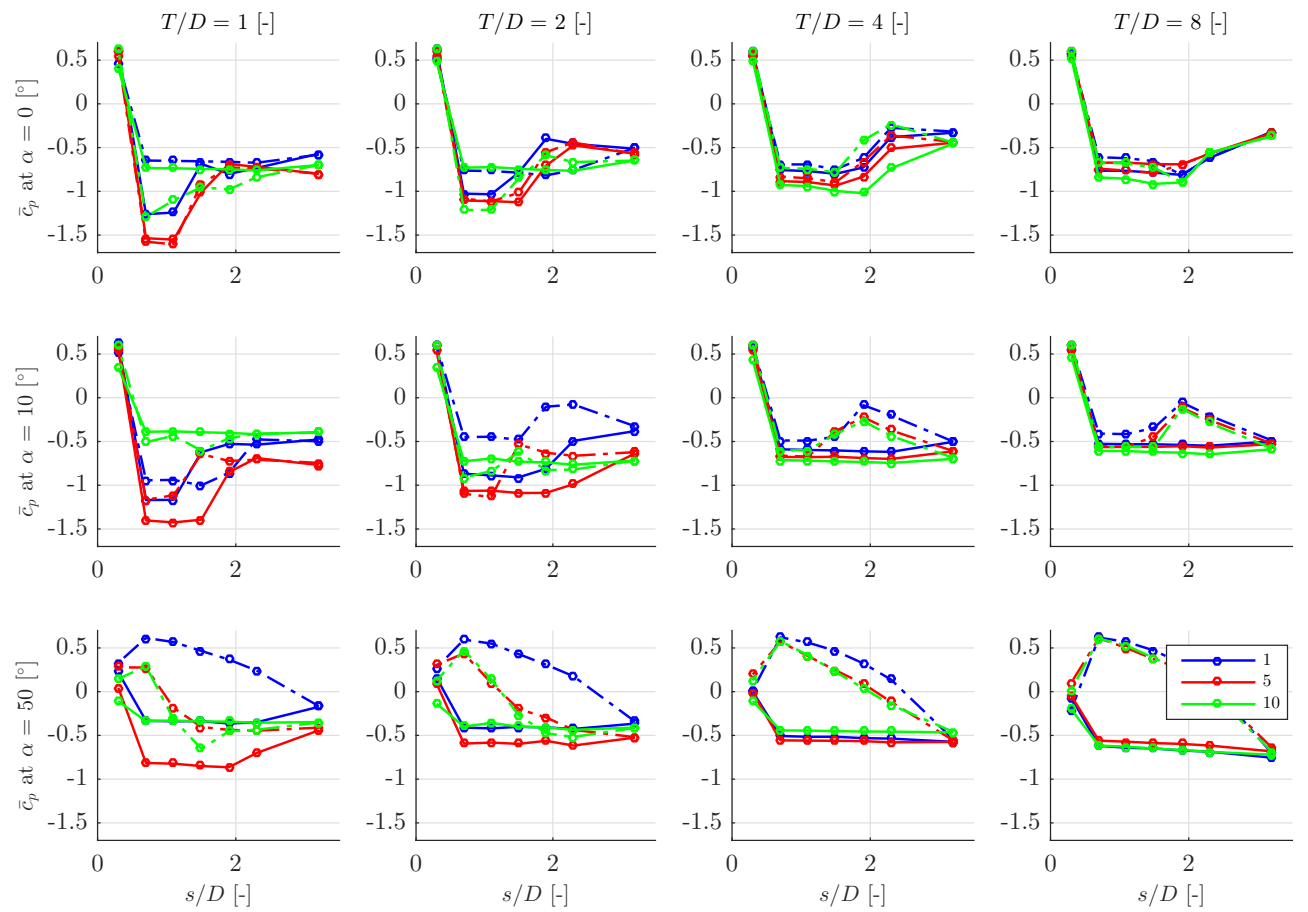

Fig. 13. Wind tunnel model: mean pressure distribution for $\alpha=0^{\circ}, 10^{\circ}, 50^{\circ}$ at $T / D=$ $1,2,4,8$ for cylinders $1,5,10$ (pressure coefficient $\overline{c_{p}}$ vs non-dimensional curvilign coordinate $s / D)$, cylinder lower side in (-) and upper side in (-.)

At $\alpha=0^{\circ}$, the mean pressure loading is symmetric on the upper and lower surfaces of cylinder 5 (central). It is also the case for the three cylinders but only when the spacing is high enough $(T / D)$, so that they are considered as alone cylinders. It is observed that the mean 
pressure coefficients are more negative (up to $\overline{c_{p}}=-1.5$ for the case $T / D=1$ ) on the central cylinder (numbered 5). The same remarks hold for the standard deviation of the pressure coefficient on this cylinder: the fluctuations are much higher than the external cylinders (numbered 1 and 10). This grid effect is important for small spacing $(T / D=1$ or 2 ) and disappears for larger values. For all wind directions, the cylinders behave as single cylinders when the spacing is above 4. The plateau observed in Figure 13 correspond to the flow separation region around the surface of the cylinder.

A quantitative comparison of the lift coefficient is presented in Table 3, where the capability of the numerical model to reproduce the flow is confirmed, except at large angle of attack. It is possible to make the link between this table and the summary Figure 11, the values of $S t \approx 0.22, \approx 0.2$ and $\approx 0.15$ of this figure match the corresponding ones of the table for the three wind directions.

\begin{tabular}{cccccc}
\hline$\alpha$ & $\begin{array}{c}\text { Wind tunnel } \\
\text { (VIV response) }\end{array}$ & $\begin{array}{c}\text { Wind tunnel } \\
\text { (lift coefficient) }\end{array}$ & CFD & $\begin{array}{c}\text { Wind tunnel } \\
\text { (lift coefficient) }\end{array}$ & CFD \\
\hline $0^{\circ}$ & 0.22 & 0.21 & 0.19 & 0.24 & 0.27 \\
$10^{\circ}$ & 0.19 & 0.20 & 0.18 & 0.20 & 0.26 \\
$50^{\circ}$ & 0.15 & 0.14 & 0.16 & 0.04 & 0.03
\end{tabular}

Table 3: Strouhal $S t$ and lift standard deviation $\sigma\left(c_{l}\right)$ for cylinder 5 depending on $\alpha$ : comparison experiment (Wind tunnel model VIV response and lift coefficient) - CFD

\section{Discussions}

This section discusses the main experimental and numerical findings presented above. The comparison of in situ and wind tunnel measurements is based on the level of turbulence of the incoming flow. A detailed analysis of the vortex shedding process of the cylinder in grid arrangement is presented. Finally, two mitigation techniques are proposed, tested and classified on the basis of their effectiveness to reduce vibration and acoustic emissions.

\subsection{Comparison of the approaches}

Figure 14 gathers the VIV responses measured in situ and in the wind tunnel. The damping independent form is presented here to focus on the VIV-grid phenomenon only. The effect of direction has been studied in the previous section: a factor of 5 between $0^{\circ}$ and $50^{\circ}$ is observed in accordance with Figure 10

The VIV curves corresponding to in situ and wind tunnel at $\alpha=50^{\circ}$ are shown in Figure 14. They reach the same maximum value of $\zeta \ddot{x}_{r m s} \approx 0.2$. The correspondency between these two curves is justified by the wind direction measurement during the important VIV events on site, taking place when the wind direction reaches $50^{\circ}$ (see Figure 7). In addition, the analysis of the turbulent content of the wind as a function of its direction is presented in Figure 15. It is observed that the wind at $50^{\circ}$ has the lower turbulent intensity $\left(I_{u}\right.$ and $I_{v} \approx 5 \%$ ), and hence is more prone to develop VIV than other wind directions for which $I_{u}$ and $I_{v}>10 \%$ (Hansen, 2007).

The difference in the size of the lock-in range measured in situ and in the wind tunnel is important. It cannot be explained by the value of the structural damping which is twice lower 
in situ than on the wind tunnel model. An alternative explanation is based on the change of wind direction taking place in situ. This fluctuation of $\alpha$ impacts the Strouhal number $(S t(\alpha)$ see figure 11) and hence the critical wind speed at which VIV starts to occur. Assuming the same size of the lock-in range (roughly $\Delta U_{r}=1.5$ ), the VIV response in situ corresponds to the enveloppe of several VIV curves corresponding to different wind direction. The situation is sketched schematically in figure 16 Note that the amplitude of the VIV response is not taken into account in the present discussion since it depends on the value of the lift coefficient, which is in turn a function of the wind direction.

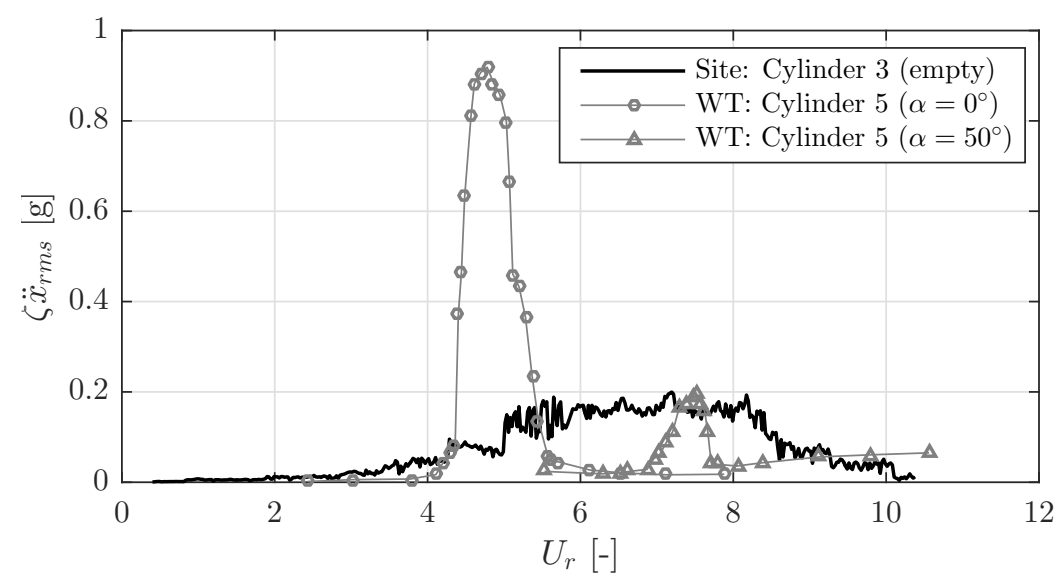

Fig. 14. VIV curve, damping independent acceleration amplitude $\zeta \ddot{x}_{r m s}$, as a function of $U_{r}$ : comparison between in situ (cylinder 3) and wind tunnel (cylinder 5 at $\alpha=0^{\circ}$ and $50^{\circ}$ )

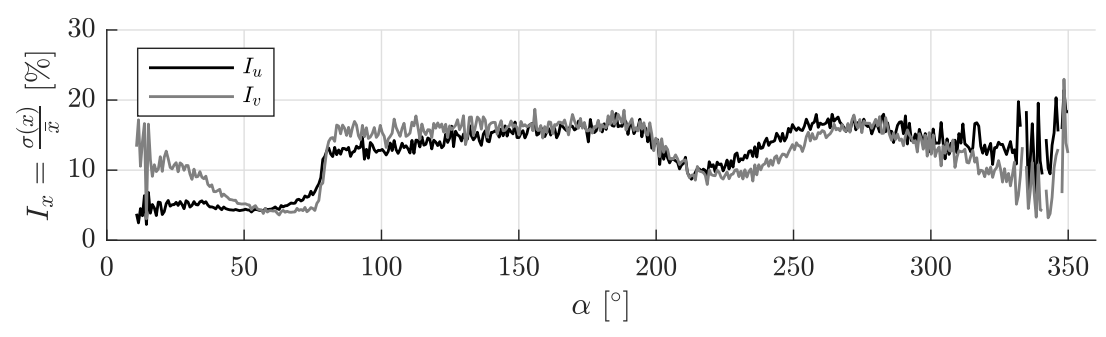

Fig. 15. In situ measurements: turbulence intensity (10 s windows) vs. wind direction

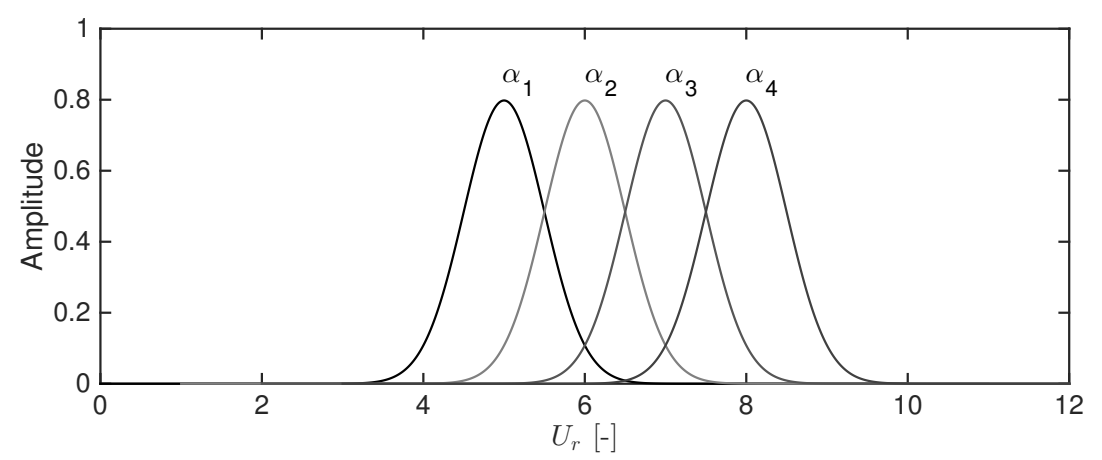

Fig. 16. Sketch of the VIV response on site as the enveloppe of multiple VIV responses 


\subsection{Grid effect on vortex shedding}

By measuring simultaneously the pressure distributions on adjacent cylinders $4,5,6$, the vortex shedding behaviour can be studied in term of phase and synchronization between cylinders. Figure 17 shows the time variation of the pressure $c_{p}$ at corners of cylinders 4 to 6 for $\alpha=0^{\circ}$ and $T / D=1,2,4$.

For small spacing $(T / D=1)$, a single vortex street region, such as commented by Zdravkovich (Zdravkovich, 1985) is observed. The upper side of cylinder 5 (in blue) and the lower side of cylinder 6 (in magenta) are in phase. The lower side of cylinder 5 (in red) and the upper side of cylinder 4 (in green) are together in phase, but out of phase with the above mentioned blue and magenta curves. Cylinders are so close to each other and interfering strongly, that the symmetric sides of two adjacent cylinders are synchronized in term of vortex shedding (redgreen or blue-magenta). The flow is separated everywhere but the pressure for the upstream corner (plain line in Figure 17) is different from the one at the downstream corner (dashed line in Figure 17). This corresponds to the step shape pressure of Figure 13 different separated flow at $s / D=0.5$ (upstream corner) and $s / D=3$ (downstream corner).

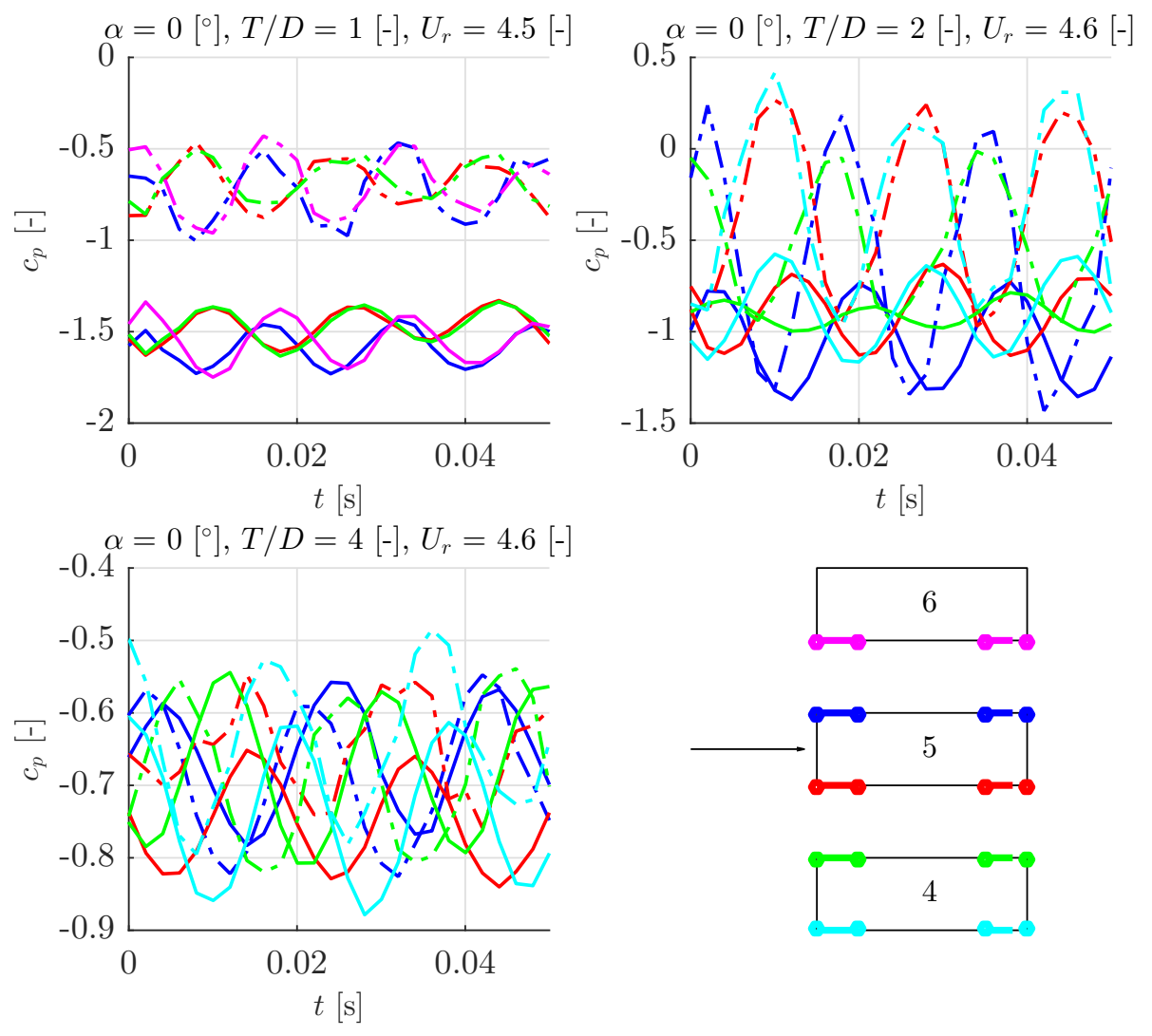

Fig. 17. Wind tunnel model: pressure $c_{p}(t)$ at corners of cylinders $4,5,6$ at $\alpha=0^{\circ}$ and $T / D=1,2,4$. Upstream $=$ solid lines and the second point $(s / D=0.5)$ on the red line in the pressure distribution in Figure 13. Downstream $=$ dashed lines and the second last point $(s / D=3)$ on the red line in the pressure distribution in Figure 13 .

For larger spacings $(T / D=2)$, a biased gap flow, or a coupled vortex street is observed (Zdravkovich, 1985). Magenta and red curves are then in phase, in opposition to blue and 
green ones. Vortex shedding is no more synchronized in alternating channels. However, the upper sides of all cylinders are in phase, the lower sides are also in phase, but the upper-lower ejection alternates, because of the spacing of the cylinders, reducing their correlation and strength. The cylinders still influence each other because the pressure at the upstream and downstream corners is still different (but less than for $T / D=1$ ). The same type of synchronization takes place for $T / D=4$, but in that case the adjacent cylinders do not influence each other. The pressure at the upstream corners gets closer to the one at downstream ones. Vortices are alternatively ejected between the upper and lower cylinder sides.

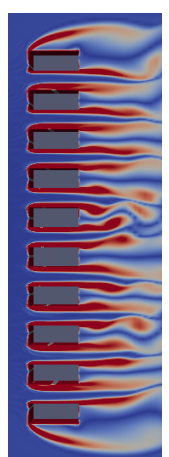

(a) $\alpha=0^{\circ}, T / D=1$

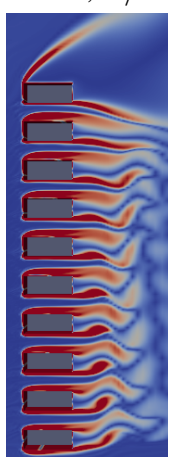

(e) $\alpha=25^{\circ}, T / D=1$

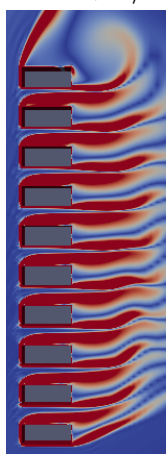

(i) $\alpha=50^{\circ}, T / D=1$

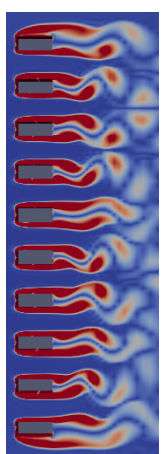

(b) $\alpha=0^{\circ}, T / D=2$

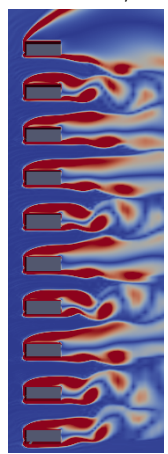

(f) $\alpha=25^{\circ}, T / D=2$

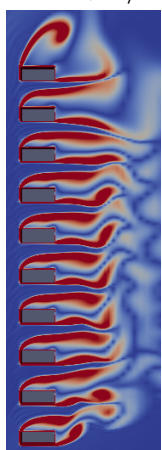

(g)
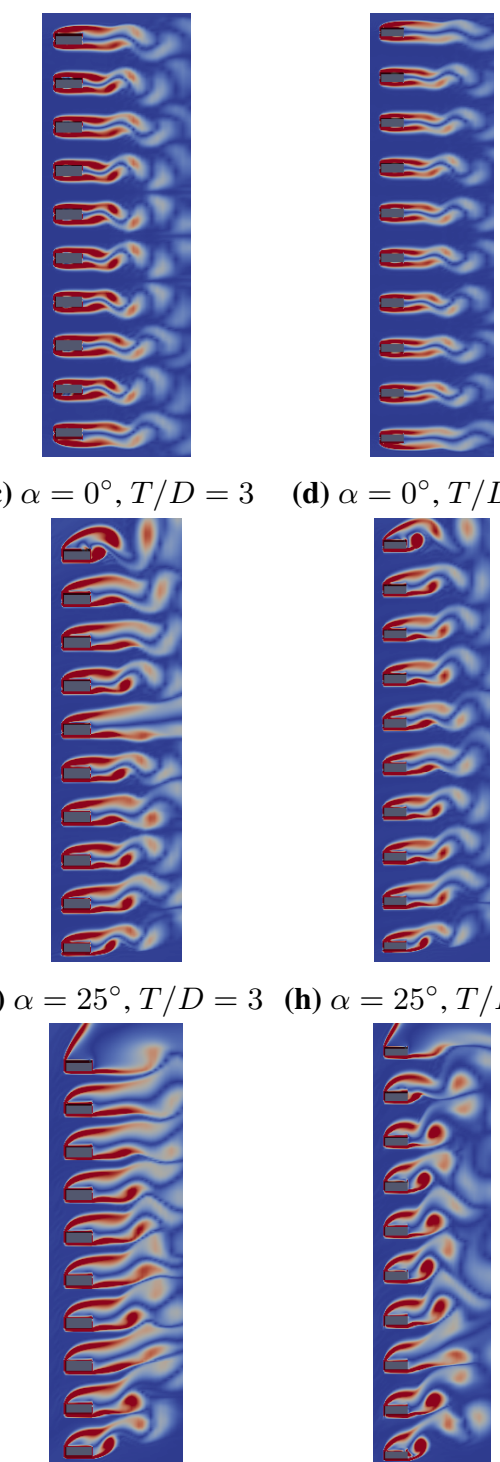

(c) $\alpha=0^{\circ}, T / D=3$

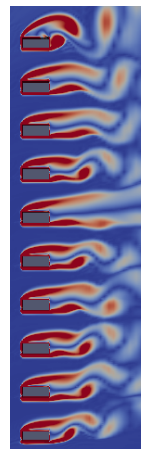

(d) $\alpha=0^{\circ}, T / D=4$

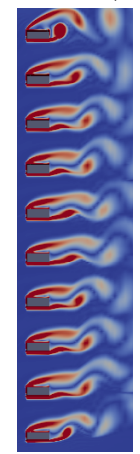

(h) $\alpha=25^{\circ}, T / D=4$

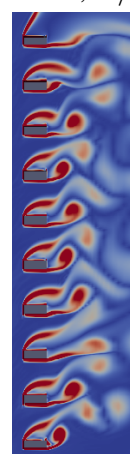

(j) $\alpha=50^{\circ}, T / D=2 \quad$ (k) $\alpha=50^{\circ}, T / D=3 \quad$ (l) $\alpha=50^{\circ}, T / D=4$

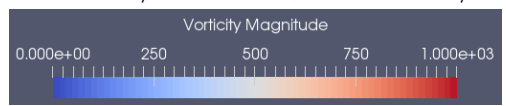

Fig. 18. CFD results - vorticity magnitude around the grid at $U=5 \mathrm{~m} / \mathrm{s}: \alpha$ and $T / D$ effect

Figure 18 shows instantaneous vorticity fields for a grid of 10 cylinders at $\alpha=0^{\circ}, 25^{\circ}, 50^{\circ}$ 
and $T / D=1,2,3,4$. The following observations are made about the spacing of cylinders from these snapshots: (i) for $T / D=1$, the flow in the grid is governed by strong shear layers and the vortices are not clearly formed one chord downstream the cylinders. Strong interactions are observed in the wake of the cylinders. (ii) For $T / D=2$ the formation of vortices is more noticeable than $T / D=1$ and their interaction is important. (iii) For $T / D=3$ and 4 , the spacing between cylinders is such that the vortex shedding process is similar to the one of a single cylinder. Other observations are made about the wind direction: (i) for $\alpha=25^{\circ}$ the vortex shedding process is disorganised, except for the case of large spacing $(T / D=4)$. (ii) For $\alpha=50^{\circ}$, strong wake interaction is observed for $T / D=1$ and 2. The end-effects are more pronounced for these spacings. A clear wake organisation is observed in the central part of the grid. (iii) For $T / D=3$ and 4 , the wake interaction decreases and typical vortex shedding phenomenon is observed.

\subsection{Mitigation techniques}

On the basis of the fundamental understanding of the grid VIV phenomenon, it is possible to propose different solutions to mitigate the vibration and acoustic effects. The incidence $\alpha$ is not anymore a parameter but depends on the atmospheric wind, hence it is a stochastic variable. With the objective to keep the grid unchanged, the parameter $T / D$ is also fixed equal to 2 . Because the grid is geometrically unchanged, the variation of the Strouhal number with wind direction is unchanged and the only non-dimensional number to vary is the Scruton number $S c$. The objective is to reduce the vibration of the phenomenon or to push it further for statistically rare wind velocities. The target is to avoid any vibration in the range $0-10$ $\mathrm{m} / \mathrm{s}$, which represents the more probable wind speeds in Belgium.

Two solutions have been implemented and tested:

1. Solution 1: Installing an horizontal beam riveted to all cylinders;

2. Solution 2: Filling cylinders with sand.

The first solution has the advantage to increase slightly the frequency of the mode which is responsible for the grid-VIV instability $(60 \mathrm{~Hz}$ to $66 \mathrm{~Hz})$. In addition, damping is added to the system by the friction of the rivets on the cylinders (see picture in Figure 19). Another effect of the beam is to distribute the energy of the excitation over several modes (at least 3 ), as can be seen from the 3 high peaks in the FFT of the bold black curve in Figure 20. This spreading is interesting in order to avoid to concentrate all the energy on a single mode, as seen in the FFT's of the initial and solution 2. The horizontal beam has also a function of dashpot: as VIV does not take place simultaneously on all cylinders, the neighbour cylinders can act as a mass damper system limiting the excitation at the location where the vibration is maximal. The second solution takes advantage of the added mass of the structure $\left(m_{S}\right)$ and added damping of the medium (sand). Both solutions increase the Scruton number. The former has the disadvantage of visual modification, while the latter increases strongly the total weight of the structure $(+200 \%)$.

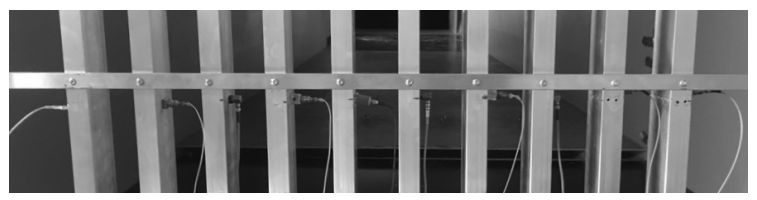

Fig. 19. Solution 1: small riveted horizontal beam (Aluminium)

Figure 20 shows the modal properties of the wind tunnel model with the two solutions implemented, in comparison with the initial case. It is shown that the first solution doubles 
the structural damping, while second one increases it by a factor of 5 . Taking into account the added mass of each solution, the Scruton number reach 45 and 225 for solution 1 and 2 respectively. The Scruton number of the initial configuration is equal to 18 .
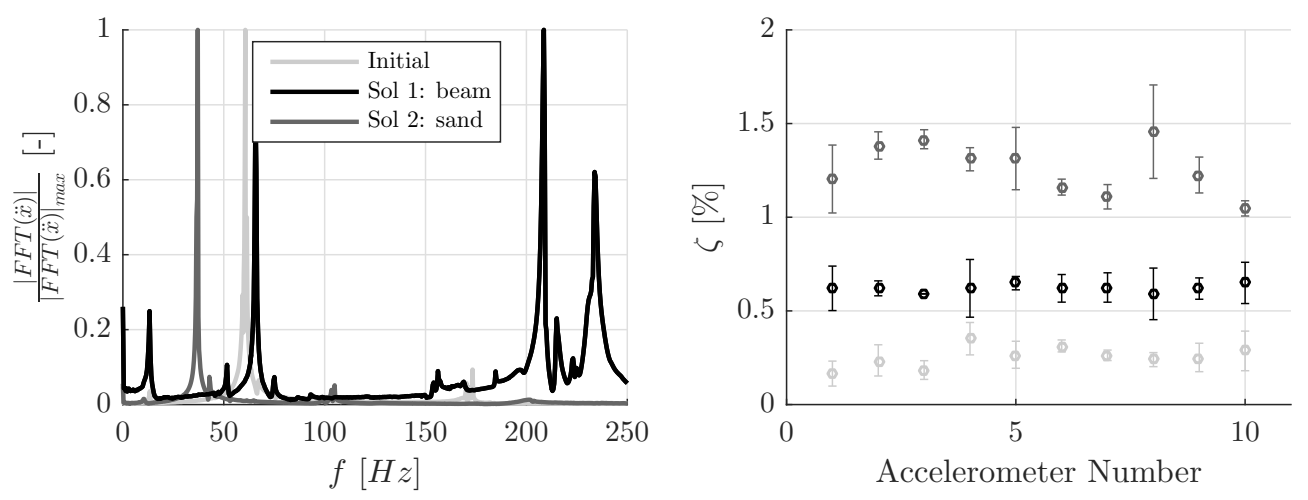

Fig. 20. Modal properties of the wind tunnel model $(U=0 \mathrm{~m} / \mathrm{s}$, cylinder 5): comparison of the initial structure with two solutions

The upper plots of Figure 21 show the acceleration of the central cylinder for the three different configurations. A small VIV response is measured for solution 2 (cylinders filled with sand). The ratio between the highest acceleration of the initial and solution 2 is equal to 6 , while the ratio of the corresponding Scruton numbers is equal to 12 . This fact might be explained by the VIV grid effect, together with an unknown variation of the sand distribution in the cylinders. The wind speed at which VIV takes place is equal to $4 \mathrm{~m} / \mathrm{s}$, as expected from the lower frequency of the filled cylinders due to added mass.

The response observed at $15 \mathrm{~m} / \mathrm{s}$ for the solution 1 has a typical clutch VIV shape. Nevertheless, it appears at a lower critical speed (ratio 2.5) than the one predicted by the ratio of frequency of 3.2. For this case, the prediction of critical galloping velocity is about $17.5 \mathrm{~m} / \mathrm{s}$, below the critical VIV speed of $19 \mathrm{~m} / \mathrm{s}$. A complex VIV-galloping interaction is triggered, known as a quenching (Mannini (Mannini et al., 2014)) with vibration beginning at a lower speed than the critical VIV prevision. Moreover, the first mode (at $66 \mathrm{~Hz}$ ) is not excited. From our explanation relying the effect of horizontal beam, it creates a link between cylinder. The non-synchronous feature of VIV on cylinders is used so that the neighbour cylinder act as a damper. This is very effective on first bending mode because beam is placed at mid-height, where vibration and mitigation is maximal.

The lower plots of Figure 21 show the acoustic intensity $I_{a}$ measured by a microphone located in the flow field inside the test section of the wind tunnel. Absolute acoustic levels are not representative of reality because of the multiple sound wave reflections on the metallic walls of the test section. For this reason we focus here on the relative differences between the three configurations. As expected from the previous analysis of accelerations, the acoustic emission is much lower when mitigation solutions are installed: a decrease of $20 \mathrm{~dB}$ is reported for $\alpha=0^{\circ}$ and only $10 \mathrm{~dB}$ for $\alpha=50^{\circ}$. Note that the linear increase of the acoustic intensity is due to the turbulent flow signature of the grid, which is different from the monoharmonic sound emission due to the VIV-grid phenomenon. It is interesting to note that the sound felt in situ was higher than the one measured in wind tunnel. This can be explained by the presence of the terrace downstream the grid, which plays a role. Indeed the frequency of vibration is $f=60 \mathrm{~Hz}$, the speed of sound is $a_{0}=340 \mathrm{~m} / \mathrm{s}$, hence the associated wavelength 
is $\lambda=a_{0} / f=5.6 \mathrm{~m}$, very close of the dimensions of the terrace ( $4 \mathrm{~m}$ by $6.5 \mathrm{~m}$ in Figure 2 ). It acts like a soundboard and amplifying the acoustic intensity, the noise experienced is felt stronger. Moreover, the terrace rear wall is made of glass, increasing this effect.
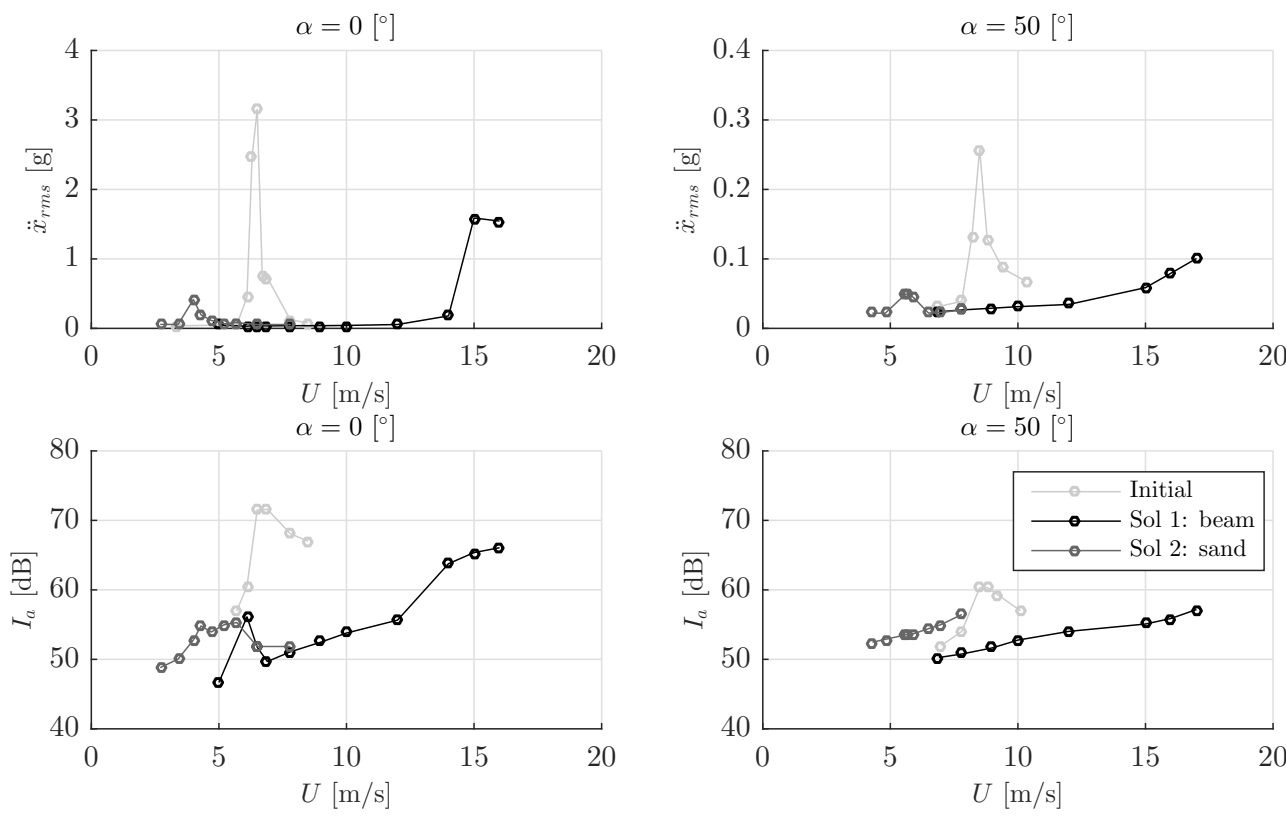

Fig. 21. Wind tunnel model: VIV curves $\ddot{x}_{r m s}$ and acoustic intensity $I_{s}$ vs $U$ at $\alpha=0^{\circ}-50^{\circ}$ for the initial and solution models

On the basis of the analysis of the two mitigation techniques, it is concluded that the adjunction of the horizontal beam leads to better performances to cancel out the VIV grid phenomenon.

\section{Conclusion}

In this work, undesirable noise occurring on a grid made of rectangular cylinders motivated the study of the VIV-grid phenomenon. Based on in situ measurements, the wind conditions leading to this instability were studied: the dominant South-West wind of Belgium at $U=5$ $\mathrm{m} / \mathrm{s}$ produces high vibration amplitudes of the cylinders. A full scale wind tunnel model of the real grid was built to reproduce the real conditions and to study more configurations, not constrained by wind conditions on site. A parametric study on the wind incidence and the cylinder spacing was carried out, highlighting the effect on the flow around a grid arrangement. The grid arrangement has a tendency to decrease the critical velocity. The wind incidence has also a strong effect on the VIV grid phenomenon: the Strouhal number decreases when wind incidence increase. The experimental pressure analysis was useful to understand the vortex shedding process responsible of the VIV-grid phenomenon. The use of the CFD model allowed to study in depth the flow around the cylinders and the coupling/synchronization of vortices. The superposition of VIV curves in the wind tunnel pointed out the driving parameters of the VIV-grid phenomenon: coupling of wind incidence, magnitude and turbulence intensity. The combination of the three different approaches allowed to have a complete understanding of the phenomenon. Finally, two mitigation techniques were proposed and tested to cancel out the aeroelastic instability and corresponding acoustic emission within the range of most likely wind speeds. 


\section{Acknowledgments}

The authors would like to thank the company V2i for providing technical support and devices necessary to make all modal and in-situ measurements.

\section{References}

Eurocode, 1991. en 1991-1-4: Actions on structures-general actions-wind actions. european committee for standardization.

H. Bergh and H. Tijdeman. Theoretical and experimental results for the dynamic response of pressure measuring systems. 1967.

R. Blevins. Flow Induced Vibrations. Krieger Publishing Co., Florida, 1990.

C. Choi and D. Kwon. Determination of the strouhal number based on the aerodynamic behavior of rectangular cylinders. Wind and Structures, 3(3), 209-220, 3(3):209-220, 2000.

S. Hansen. Vortex-induced vibration of structures. In Structural Engineers World Congress, Bangalore, India, 2007.

C. Knisely. Strouhal numbers of rectangular cylinders at incidence: A review and new data. Journal of Fluids and Structures, 4(4):371 - 393, 1990. ISSN 0889-9746. doi: https://doi. org/10.1016/0889-9746(90)90137-T. URL http://wwW.sciencedirect.com/ science/article/pii/088997469090137T

C. Mannini, A. Marra, and G. Bartoli. Viv-galloping instability of rectangular cylinders: Review and new experiments. Journal of Wind Engineering and Industrial Aerodynamics, 132:109 - 124, 2014. ISSN 0167-6105. doi: https://doi.org/10.1016/j.jweia.2014. 06.021. URL http://www.sciencedirect.com/science/article/pii/ S0167610514001299.

H. Nakaguchi and S. Muto. An experimental study on aerodynamic drag of rectangular cylinders. Journal of Japan Aeronautics Space Science, 16:1-5, 1968.

A. Okajima. Strouhal numbers of rectangular cylinders. 123:379-398, 1982.

M. Ong. Unsteady rans simulation of flow around a 5:1 rectangular cylinder at high reynolds numbers. 5, 2013.

F. Rigo, V. Denoel, and T. Andrianne. Vortex induced vibrations of rectangular cylinders arranged on a grid. Journal of Wind Engineering and Industrial Aerodynamics, 177:1 13, 2018. ISSN 0889-9746. doi: https://doi.org/10.1016/j.jweia.2018.04.011.

A. Techet. Vortex Induced Vibrations. MIT, Boston, 2005.

M. Zdravkovich. Flow induced oscillations of two interfering circular cylinders. Journal of Sound and Vibration, 101:511-521, 1985. 